\title{
Clinical Case: An Integral Approach from the Bioethical Perspective to the Disorders of Sexual Development (DSD)
}

\author{
Freddy Andrés Barrios Arroyave* \\ Department of Community Health, GISCO group investigator, Fundación Universitaria Autónoma de las Américas, Colombia
}

*Corresponding author: Freddy Andrés Barrios Arroyave, Master in Sexual and Reproductive Health, El Bosque University Department of Community Health, GISCO group investigator, Fundación Universitaria Autónoma de las Américas, Colombia.

Submission: 湭 October 26, 2017; Published: 監January 09, 2018

\begin{abstract}
Disorders of sex development (DSDs) can be distressing conditions for parents of newborns suffering from this condition. This alteration in sexual differentiation is much more frequent than it appears and primary health care workers need to be trained to guide parents and family members in making informed decisions to overcome these problems in the field of human reproduction. This is a case of a newborn with ambiguous genitalia who was valued by an interdisciplinary team although there were difficulties in choosing the best decision-making.
\end{abstract}

Keywords: Disorders of sex development (DSDs); Sex differentiation; Decision making; Ethics, medical; Bioethical issues

\section{Clinical Data}

This is the case of a newborn son of a 26-year-old primigravida woman. Pregnancy was apparently normal, nevertheless prenatal control results are unknown. The woman had a vaginal delivery at term (41 weeks) and no complications were mentioned. In the first evaluation the doctor observed that the newborn has male genital appearance; however, the mother notices that the urethral opening is at the base of the phallic axis and the phallus is short. It is evaluated by a pediatrician, who, based on the doubts of the mother, suspects the diagnosis of congenital adrenal hyperplasia (CAH). The physician provides explanations to parents about the findings and possible condition of the newborn, as well as information about the controversies regarding to gender allocation, parenting, and early surgery. An independent pediatric urologist examines the newborn and concludes that the baby should be a virilized girl and therefore considered as a real male child; so he resolves the case in this way: the surgical procedure of reassignment of sex must be consider. Meanwhile the pediatrician indicates that the result of the ultrasound performed to the newborn reports a formed uterus and presence of ovaries. A sample of umbilical cord blood is taken to evaluate the karyotype, but its result is not mentioned. The parents, their personal friends and the relatives have doubts about the appropriate time to schedule the hypospadia correction surgery; to which the doctor has no satisfactory answer for them.

\section{Considerations}

Based on the principles that govern clinical ethics, Jonsen [1] proposes that there are some questions that must be answered or clarified by the professional or the team of professionals who face decision making. Thus, medical indications should be primarily governed by the principles of beneficence and non-maleficence. From this perspective, it must be taken into account that this is a newborn diagnosed with a probable Disorder of Sex Development (DSDs), possibly a virilising congenital adrenal hyperplasia (HSC), in which the objectives of the surgical treatment would be to correct the appearance of the genitals (surgical reassignment of the anatomical sex) and to improve the urogenital function.

First it is important to note that this is not a medical emergency, since there is no risk of urogenital malignancy, infection or acute cardio respiratory or metabolic decompensation, hence its resolution and decision regarding to the allocation of phenotypic sex can be deferred. And secondly, according to the American Academy of Pediatrics, it is relevant to have the result of karyotype in order to make a more precise diagnosis of DSDs to achieve more accuracy and provide a correct treatment. In addition, other paraclinical exams such as blood glucose, blood electrolyte levels, among others might be necessary [2-4].

Also, regarding the patient preferences, the principle of respect for Autonomy must be considered. For the case, it is obvious that the patient does not have mental or legal abilities to decide for herself, so she would be incompetent to make the decision to undergo medical and surgical treatment. Her parents, who serve as her legal representatives and those in charge of the newborn, must be well informed about the terms of the diagnosis and treatment 
options; as well as receiving (on the part of the multidisciplinary health team that must always deal with these cases) information and complete advice on the foster sex and the pros and cons of eventual hormonal and surgical treatments [5-10].

Another important aspect to consider is the quality of life for the patient, in which the principles of beneficence and non-maleficence must be also observed, and respect for personal autonomy. In this sense, it should be considered that if the surgical procedure in question is performed, in the future the patient's chances of having adequate sexual and reproductive functionality are high (although, of course, without any certainty) possibly contrary to what would happen if the treatment is ignored. On the other hand, it is not clear that the possible physical, mental and social problems that may be developed in the future may be reduced or, aggravated by treatment. Some authors consider that this will depend very much on the socio-cultural context of the family and the environment, the foster sex that is assigned and the imaginaries about his/her own body as well as the experience of the sexuality that later develops this person [5-10].

According to Jonsen [1], contextual features are also an important point for making decisions, in this case principle of Justice must respected. Although it is not mentioned in the case report as such, it must be consider whether there are family, professional, religious or cultural factors that may influence treatment decisions. Also, limits to confidentiality must be taken into account. In this case it is extremely important to question this aspect because it can be seen from the first moments of the manifestation of the problem, that there are already some parents' friends and relatives aware about the issue in addition to the parents and the health staff, which can violate the confidentiality that the situation deserves.

However, confidentiality does not only mean that people outside the situation make decisions or find out about intimate situations that should be resolved exclusively by the parents, it also has to do with the discretion that the health professionals in charge should have in relation to the care and the reservation of the clinical history, to the extent that this may violate the regional legal framework of the doctor-patient/family relationship. Likewise, the professional who has some type of conflict of interest should also be clearly expressed in order to take the case to clinical teaching or for the purposes of research or scientific publication $[5,6,11]$.

\section{Analysis and Discussion}

For the treatment of DSDs, and in this particular case, it is important to recognize several fundamental aspects:

A. Medical intervention in sexual development divergences or Disorders of Sex Development (DSDs) requires a multidisciplinary approach, not only from the medical (general medicine, pediatrics, urology, gynecology, etc.) and legal point of view, but also with participation from psychology, liaison psychiatry, social work, among other professionals. On the other hand, it should be borne in mind that the complexity of these cases requires that decisions be individualized in each situation.
B. According to Hughes \& Mejias [5,6,11] "DSDs can be considered as congenital conditions in which there is a discrepancy between the sex definition criteria: chromosomal, gonadal, genital, phenotypic or morphological and psychosocial, and whose development is atypical". With respect to the available classifications reviewed in the same article, the case could be reasoned within the category of Congenital Development of ambiguous genitalia (eg virilizing congenital adrenal hyperplasia, 46 , XX, clitoromegaly, micropenis, etc.); so that for this case it would be opportune to obtain the genotypic sexual characteristics of the patient by performing a karyotype to get a correct classification of the disorder.

C. There are several treatment options, each one with advantages and disadvantages, which must be analyzed in the light of the principles that govern professional medical ethics: beneficence, non- maleficence, justice and autonomy.

In this sense, the paternalistic or "dominant" model consists of the early assignment of anatomical sex and social gender, through surgical genital remodeling and the prescription of hormonal treatment before the age of three years. This model, based on the experiences and theories of John Money from the Johns Hopkins Hospital workgroup, argues that gender identity, as a sociocultural construction, overlaps biological and anatomical sex; that is, the gender identity in children develops according to the role of foster sex, while it coincides with the appearance of the genitals. Therefore, it was considered possible to reassign sex to patients with DSDs before that age [11].

From another perspective, the model of "full consent", which is based on respect for autonomy, non-maleficence and the promotion of free personality development, recommends deferring surgical treatment and hormone replacement therapies until the patient has enough capacity to participate adequately in the informed decision making about his/her gender identity and genital remodeling. This model takes into account the possible psychological adverse effects of surgical reassignment treatment in the future, as well as the biopsychosocial complexity represented by gender identity. In this way, it is considered to defer this type of procedure until full consent can be obtained from the minor, except in case of emergency situations (risk of malignization, risk of infection, etc.). Thus, it is advised that for minor interventions the child must be approximately 5 or 6 years old and for more complex interventions they could be between 12 and 14 years old [11].

The "right balance" or "fair middle" model arises from theories that suggest that neither the dominant protocol nor the "full consent" correctly meet all the principles to promote the best benefit for patients. This perspective explains that the model of "full consent", in which the will of the minor patient is fundamental for decision making, in some cases can generate a significant psychological trauma for the child or adolescent and his/her family. For these reasons, this stream promotes a "family-centered" protocol of consent based on counseling, education and support processes provided by a multidisciplinary team of experts [7,9-11]. 
An option of complementary treatment, is the one that was proposed by the consensus of the American Academy of Pediatrics on 2006, which mentioned the need for gender allocation for all patients, previous careful evaluation of the case by the institution's multidisciplinary committee of experts, which must "take into account clinical aspects such as appearance genitalia, chromosomal sex, future reproductive possibilities, the specific nature of each condition, endocrine aspects, as well as family and cultural considerations [7,9-11]. The consensus emphasized the idea that gender allocation cannot be based exclusively on the appearance of the external genitalia.

The management guides of the Texas Children's Hospital workgroup suggest that gender assignment is necessary at the neonatal stage, but that it does not necessarily require emergency medical or surgical intervention. Gender allocation, especially for parenting purposes and for the psychosocial and cultural role, should be done by the parents, and it is again emphasized that careful multidisciplinary consultation with a clinical team of experts should be done. This team should accompany the family and the patient throughout childhood and during the period of transition into adulthood (adolescence).

In some Latin American countries such as Colombia, there is a legal framework to deal with the management of patients with DSDs. Judgment SU-337 of 1999 states that when the child has a DSDs and is younger than 5 years, he or she does not have sufficient capacity to give informed consent for the intervention nor has reached the threshold of consolidation in his/her sexual identity, so the decision may be made by the parents (substitute future-oriented consent), provided that it is informed, qualified and persistent consent, in accordance with medical recommendations [7,8]. This recommendation is based on the principle of family privacy.

If the child has a DSDs and is older than 5 years, there is usually no need for urgent intervention, so that the surgical option or hormonal therapy can be deferred until the patient is able to express their consent, which has special characteristics, since it would be an "informed consent", which is composed of the will of the child, and influenced by the concept of their parents and the expertise of the multidisciplinary committee of experts, who should privilege the principles of respect for Autonomy and the free development of personality. This requires the establishment of a complete advisory protocol and permanent accompaniment, including the possibility of a stepwise authorization.

\section{Case Resolution}

Considering all of the above, and applying the principles of medical ethics, it is considered that the most appropriate approach for this case, which does not constitute medical urgency, is to balance the models of full consent, the guidelines of the Texas Children's Hospital, the consensus of experts of the American Academy of Pediatrics and the concepts that emerge from legal frameworks of some countries as those issued by the Colombian Constitutional Court and to take from each one the pertinent considerations that allow to solve the case.
In this way, it is possible to conciliate an agreement among the recommendations in scientific literature, the consensus of the parents and the outcome of the evaluation of the case by the multidisciplinary expert committee, who must provide a complete, informed and unbiased assessment to both: the family and the professional in charge, besides an accompaniment in the time, that includes not only the childhood but the adolescence.

Thus, taking into account that it is a baby with a formed uterus and ovaries (has female internal genitalia) confirmed by ultrasonographic screening, the result of the karyotype is considered important to complement the diagnosis. Having a possible female karyotype $46, \mathrm{XX}$ and with the evidence of female internal genitalia and ambiguous external genitalia, parents could now assign the female sex for purposes of parenting, as well as socio-cultural role.

The surgical procedure of genital remodeling (possible vaginoplasty) as well as the accompanying hormonal treatment, would be differed for when the patient has a formation of imaginary about her role of social gender and about her sexual identity, that allows the informed taking of the decision, this could happen in a range between eight and twelve years old, or even earlier. However, it is reiterated that all this must be subjected to a critical judgment by the committee of experts who would advise the case.

\section{References}

1. Jonsen AR, Siegler M, Winslade WJ (2010) Clinical Ethics: A Practical Approach to Ethical Decisions in Clinical Medicine, ( $7^{\text {th }}$ edn), (Lange Clinical Science collection). McGraw-Hill Medical, USA.

2. Hutson, John M, Warne, Garry L, Grover, et al. (2012) Disorders of Sex Development An Integrated Approach to Management. ( $1^{\text {st }}$ edn), Springer-Verlag Berlin Heidelberg, Germany.

3. Kim KS, Kim J (2012) Disorders of Sex Development. Korean J Urol 53(1): 1-8.

4. Hughes IA (2008) Disorders of sex development: a new definition and classification. Best Pract Res Clin Endocrinol Metab 22(1): 119-134.

5. González P, Velásquez C, Duque SP (2010) Legal issues of intersexual states. The Colombian case. Iatreia. University of Antioquia. Medellin Colombia 23(3): 204-211.

6. Pinto BJ, Guzmán F, Moreno SE (2013) Medical ethics and clinical bioethics: some general considerations. Rev Medica Sanitas 16(2): 3437.

7. Velasquez C, González P, Sarmiento IC (2007) Principles and rights involved in the legal analysis of intersexual states in minors in Colombiathe case of hermaphroditism. Estud, Faculty of Law and Political Sciences. University of Antioquia. Medellín, Colombia, USA.

8. Pinto BJ (2010) Bioethics criticism and society: beyond the principialism. Colombian Journal of Bioethics, Universidad el Bosque, Colombia, 5(1): 73-84.

9. Öçal G (2011) Current Concepts in Disorders of Sexual Development. J Clin Res Pediatr Endocrinol 3(3): 105-114.

10. Lee PA, Nordenström A, Houk CP, Ahmed SF, Auchus R, et al. (2016) Global Disorders of Sex Development Update since 2006: Perceptions, Approach and Care. Horm Res Paediatr 85(3): 158-180.

11. Pinto BJ, Gulfo R, Mojica MC, Endo JH (2013) Phallus or Fallopian? Informed consent, equivocal anatomy and divergences of sexual development: medical, legal and bioethical aspects. Colombia Journal of Bioethics 8(2): 64-86 